BLS 32, No 1 2006. DOI: http://dx.doi.org/10.3765/bls.v32i1.3476 (published by the Berkeley Linguistics Society and the Linguistic Society of America)

\title{
Frame-Based Constraints on Lexical Choice in Metaphor
}

\author{
KAREN SULLIVAN \\ University of Queensland
}

\section{Introduction}

Most of what we know about conceptual metaphors like KNOWING IS SEEING and HAPPINESS IS LIGHT comes from metaphoric language (cf. Sweetser 1990, Kövecses 2002), yet there are some substantial gaps in our understanding of metaphoric language itself. For example, why do many semantically similar items have different metaphoric uses? Why does brilliant metaphorically mean 'intelligent', as in brilliant idea (via KNOWING IS SEEING), whereas sunny metaphorically means 'cheerful', as in sunny mood (via HAPPINESS IS LIGHT)? Both sunny and brilliant refer to qualities of light, so these items might be expected to have the same metaphoric uses and limitations. To further complicate the issue, the adjective bright can be used in either KNOWING IS SEEING or HAPPINESS IS LIGHT, as in bright idea 'intelligent idea' or bright mood 'cheerful mood'.

In this paper I hope to strip away one layer of mystery surrounding lexical choice in metaphor, using the tools of frame semantics (Fillmore 1982). I argue that the frames evoked by lexical items' nonmetaphoric senses can determine which items are chosen to express a given conceptual metaphor. I suggest that the Invariance Principle (Lakoff 1993) applies to frame structure as well as imageschema structure, and can help account for the role of frames in metaphoric extension.

My analysis is based on a study of the metaphoric and nonmetaphoric uses of a set of adjectives and adverbs in the British National Corpus. The metaphoric uses involve either HAPPINESS IS LIGHT or one of two submappings of KNOWING IS SEEING: INTELLIGENCE IS LIGHT-EMISSION or COMPREHENSIBILITY IS VISIBILITY. The nonmetaphoric senses of these modifiers evoke particular frames, which (in accordance with the extended Invariance Principle), must be carried over into the items' metaphoric uses, making them either suitable or unsuitable for expressing HAPPINESS IS LIGHT, INTELLIGENCE IS LIGHT-EMISSION, or COMPREHENSIBILITY IS VISIBILITY. This analysis will explain, among other things, why brilliant means 'intelligent' but sunny means 'cheerful', and why bright can refer to either intelligence or cheerfulness. 


\section{Karen Sullivan}

\section{Frames, domains, and the Invariance Principle}

Before diving into the data, I will briefly characterize my interpretation of how frames and domains interact. By 'metaphoric domain' I refer to the structure comprising all schematic information potentially available for mapping via a given metaphor. Much of the schematic information in a domain comes from frame structure. For example, certain structure in the BODY domain (the source domain of THE MIND IS A BODY) is derived from the *EXERCISE frame (evoked by the verb exercise), as in Figure (1). ${ }^{1}$ A domain is usually structured by multiple frames, so that for example the BODY domain is structured by frames related to 'eating' (INGESTION), 'dying' (DEATH), and others not shown in this diagram.

(1) The *EXERCISE frame structures the BODY domain

\section{*EXERCISE FRAME BOD DOMAIN}

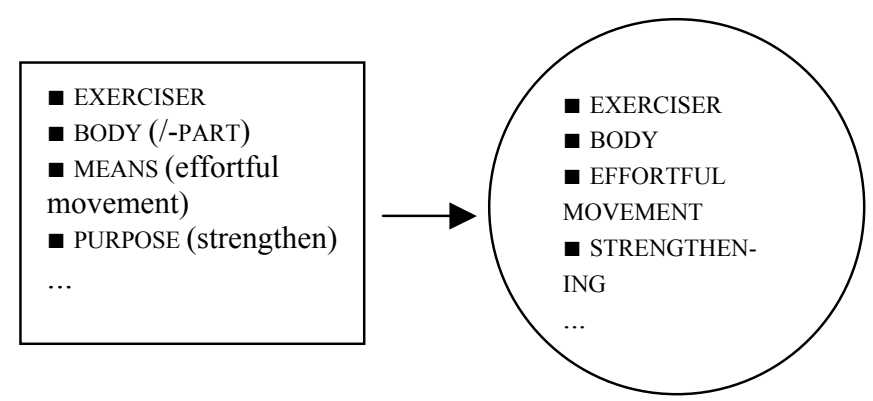

The information about 'exercise' in the BODY domain can be mapped to a target domain, such as MIND, via THE MIND IS A BODY. The mapping of 'exercise' structure to MIND is evident in expressions like mental exercise or a workout for your brain. Several mappings of THE MIND IS A BODY which preserve EXERCISE frame elements are shown below.

(2) THE MIND IS A BODY

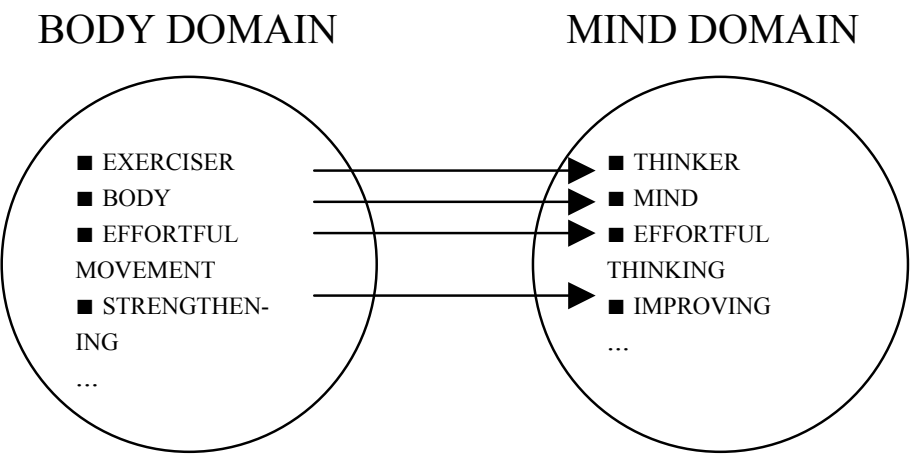

\footnotetext{
${ }^{1}$ Frames marked with an asterisk have not been documented by the FrameNet project. All frame diagrams and discussions in this paper include only a subset of the frames' structure; more complete analysis of the documented frames can be found at http://framenet.icsi.berkeley.edu/.
} 


\section{Frame-Based Constraints on Lexical Choice in Metaphor}

Metaphoric mappings preserve frame relations and inferences as well as frame elements. In the BODY domain, the BODY element must refer specifically to the EXERCISER's body. This relation carries over into the MIND domain, in which the MIND must be specifically the THINKER's mind. Likewise, the STRENGTHENING element in the BODY domain is the effect of EFFORTFUL MOVEMENT, which leads to the inference in the MIND domain that EFFORTFUL THINKING will result in mental IMPROVEMENT.

The preservation of frame elements, relations, and inferences in metaphoric mappings suggests that frame structure, like image-schema structure, is subject to the Invariance Principle (Lakoff 1993:215):

Metaphorical mappings preserve the cognitive topology (that is, the image-schema structure) of the source domain, in a way consistent with the inherent structure of the target domain.

If the definition of 'cognitive topology' is extended to include frame structure as well as image-schema structure, then the preservation of frame elements and relations in metaphors such as THE MIND IS A BODY is predicted.

The Invariance Principle does not predict whether any particular sourcedomain structure will be mapped in a given instance of a metaphor. Metaphoric mappings are always partial, preserving only a subset of the source-domain structure (Lakoff and Johnson 1980). The structure that is mapped will vary even between instances of a single conceptual metaphor, when different submappings of the metaphor are involved in each instance.

The partial nature of metaphoric mappings should be kept in mind when tracking the effects of frame structure on metaphor. Since metaphoric domains are often structured by multiple frames, different submappings of a metaphor may preserve the structure of different frames. For instance, IDEAS ARE FOOD (as evinced by phrases like half-baked ideas and other examples cited by Lakoff [1980:46-47]) is a submapping of THE MIND IS A BODY which does not map elements from the *EXERCISE frame. Instead, the submapping draws on the structure of the INGESTION frame, such that an INGESTOR maps to a THINKER, INGESTIBLES map to IDEAS, and so forth. The fact that different submappings can map material from different frames will be a crucial assumption in sections (4) and (5) of this paper, which compare the frame structure involved in two submappings of KNOWING IS SEEING: INTELLIGENCE IS LIGHT-EMISSION and COMPREHENSIBILITY IS VISIBILITY.

Much of the analysis in this paper depends on another corollary of the Invariance Principle, one that is usually assumed rather than stated: that metaphorically mapped 'cognitive topology' is evidence of source-domain structure. In other words, structure that is mapped must logically be present in the source domain. Mappings in THE MIND IS A BODY, such as EFFORTFUL THINKING IS EFFORTFUL MOVEMENT and A THINKER IS AN EXERCISER, demonstrate that EFFORTFUL MOVEMENT and EXERCISER are elements in the BODY domain, which in turn provides evidence that the frame with these elements, *EXERCISE, is structuring 


\section{Karen Sullivan}

the BODY domain. Throughout this paper, metaphoric mappings will be taken as evidence of source-domain structure, including frame structure.

Metaphorically mapped frame structure can be directly compared with the frame structure evoked by the nonmetaphoric senses of lexical items. If the hypothesis of this paper is correct, and lexical items' frame structure constrains their compatibility with a given metaphor, we will find the reasons for the items' compatibility or incompatibility in the frame structure evoked by the items' nonmetaphoric uses.

\section{Methodology}

The data were collected in a series of searches within the British National Corpus (c.100 million words) involving the following collocations: bright $N(\mathrm{n}=4,172)$, brightly $V(\mathrm{n}=323), V$ brightly $(\mathrm{n}=160)$, brilliant $N(\mathrm{n}=1,456)$, brilliantly $V(\mathrm{n}$ $=100), V$ brilliantly $(\mathrm{n}=83)$, sunny $N(\mathrm{n}=587)$, sunnily $V(\mathrm{n}=1)$, clear $N(\mathrm{n}=$ $3,524)$, clearly $V(\mathrm{n}=2,591), \operatorname{dim} N(\mathrm{n}=345), \operatorname{dark} N(\mathrm{n}=4,856)$.

The search items' metaphoric and nonmetaphoric senses were usually disambiguated by the collocated nouns and verbs (for example, brilliant idea involves a metaphoric sense of brilliant, whereas brilliant star involves a nonmetaphoric sense). When the collocated noun or verb permitted multiple senses of the modifier (as in brilliant one), I determined which sense was intended based on the larger context in which the collocation occurred.

Collocations with over a thousand hits were counted only above a certain frequency cutoff. Single-occurrence collocations were excluded (except for sunnily began, the only instance of sunnily $V$ ).

\section{Lexical choice in HAPPINESS IS LIGHT}

A chief function of several adjectives referring to 'light' is the communication of the metaphor HAPPINESS IS LIGHT. For example, the adjective bright means 'happy/cheerful', as in looking on the bright side, bright greeting and bright outlook, in $33 \%$ of the total collocations of bright. The adjectives sunny and dark also express HAPPINESS IS LIGHT as in sunny disposition or dark mood, with the frequencies shown below.

Table 1. HAPPINESS IS LIGHT collocations

\begin{tabular}{|l|l|l|l|l|l|}
\hline Item & $\begin{array}{l}\text { Total } \\
\text { 'LIGHT' }\end{array}$ & Example & $\begin{array}{l}\text { Total } \\
\text { 'HAPPI- } \\
\text { NESS' }\end{array}$ & Example & $\begin{array}{l}\text { Percent } \\
\text { 'HAPPINESS' } \\
\text { (of total hits) }\end{array}$ \\
\hline bright & $\mathbf{2 4 3 0}$ & bright place & $\mathbf{1 3 7 1}$ & bright disposition & $\mathbf{3 2 . 9}$ \\
\hline brightly & $\mathbf{3 8 2}$ & glowed brightly & $\mathbf{1 0 1}$ & laughed brightly & $\mathbf{2 0 . 9}$ \\
\hline dark & $\mathbf{4 3 4 0}$ & dark room & $\mathbf{4 4 4}$ & dark thought & $\mathbf{9 . 2}$ \\
\hline sunny & $\mathbf{5 5 4}$ & sunny terrace & $\mathbf{3 3}$ & sunny mood & $\mathbf{5 . 6}$ \\
\hline sunnily & $\mathbf{0}$ & & $\mathbf{1}$ & $\begin{array}{l}\text { sunnily began to } \\
\text { take requests }\end{array}$ & $\mathbf{1 0 0}$ \\
\hline
\end{tabular}

NOTE: The column Total 'LIGHT' includes all nonmetaphoric senses referring to 'light' or 'seeing', regardless of frame evoked. Percent 'HAPPINESS' reflects a percentage of the total analyzed collocations of the listed item, which may include senses not added into the totals for either 'LIGHT' or 'HAPPINESS'. 


\section{Frame-Based Constraints on Lexical Choice in Metaphor}

However, some adjectives referring to 'light' cannot express HAPPINESS IS LIGHT. For example, brilliant never means 'cheerful' or 'happy', as in: ?looking on the brilliant side or ?brilliant disposition.

We can see why brilliant differs from adjectives like bright and sunny when we turn to the nonmetaphoric uses of these adjectives, and specifically the frame structure that these uses evoke. Nonmetaphoric bright, sunny and dark often modify nouns denoting a location, as in bright room, sunny place, and dark corner. However, brilliant is rarely used in this way, as in collocations like ?brilliant place or ?brilliant street.

This difference is indicative of the fact that adjectives like bright and sunny usually evoke the LOCATION_OF_LIGHT frame as in Figure (3), which involves a LOCATION where the light is apparent (called a 'GROUND' element in FrameNet notation). ${ }^{2}$ The modified location nouns denote this LOCATION element.

(3) LOCATION_OF_LIGHT frame (sunny, bright, dark)

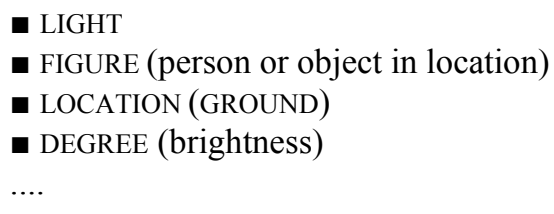

The adjective brilliant, on the other hand, typically refers to light emanating from a source, as in brilliant star or brilliant torch. These uses evoke the LIGHT_ MOVEMENT frame in Figure (4), which does not involve a LOCATION element.

(4) LIGHT_MOVEMENT frame (brilliant, bright, dim)

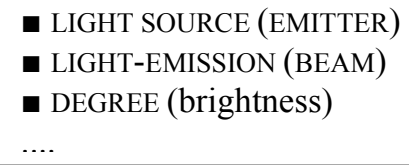

Adjectives that evoke the LOCATION_OF_LIGHT frame frequently modify nouns denoting the LOCATION element in this frame; while adjectives that evoke the LIGHT_MOVEMENT frame do not modify these nouns. This distinction makes the presence or absence of collocated LOCATION nouns a useful diagnostic of which frame an adjective evokes.

The LOCATION element also provides evidence that the LOCATION_OF_LIGHT frame is part of the LIGHT source domain. The metaphor HAPPINESS IS LIGHT includes the mapping HAPPY STATES ARE LIT LOCATIONS, apparent in preposition phrases such as in a sunny mood or in a dark state of mind. As discussed above,

\footnotetext{
${ }^{2}$ When my name for a frame element differs from the one found in FrameNet, the FrameNet term is indicated in parenthetical small caps following the element name. Clarifying descriptions of the elements are in parenthetical normal text following the element name.
} 


\section{Karen Sullivan}

metaphoric mappings reflect source-domain frame structure via the extended Invariance Principle. The mapping HAPPY STATES ARE LIT LOCATIONS (shown in boldface in Figure [5] below) therefore reflects a LOCATION element in the source-domain structure; and the presence of a LOCATION element is evidence, in turn, that the LIGHT domain is structured by LOCATION_OF_LIGHT.

(5) HAPPINESS IS LIGHT

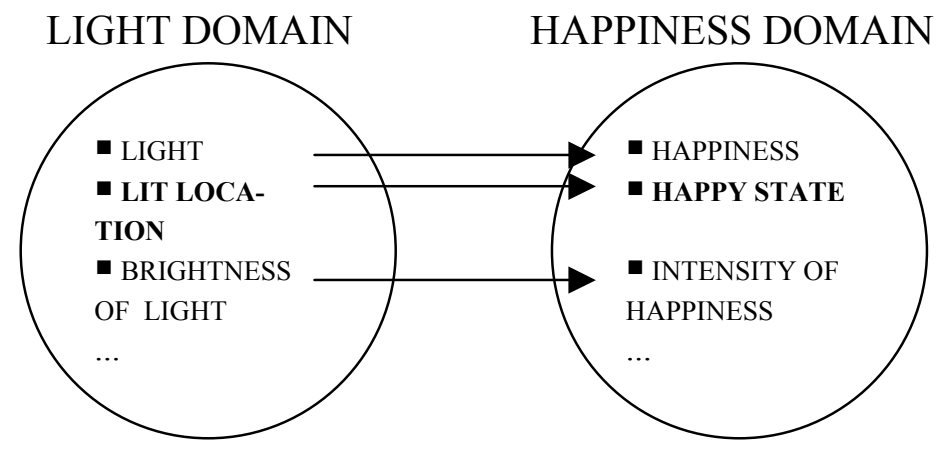

Adjectives like sunny, which evoke the LOCATION_OF_LIGHT frame, can express the metaphor HAPPINESS IS LIGHT because their frame structure matches the frame structure of the LIGHT source domain. Adjectives like brilliant, which evoke a frame other than LOCATION_OF_LIGHT, are inconsistent with the LIGHT source domain and cannot acquire metaphoric meanings in the domain of HAPPINESS. This analysis of the data in Table 1 supports the central hypothesis of this paper: that lexical items' frame evocation constrains the items' uses in metaphor.

\section{Lexical choice in INTELLIGENCE IS LIGHT-EMISSION}

Although the frame structure of brilliant renders it incompatible with the metaphor HAPPINESS IS LIGHT, this same frame structure evidently permits brilliant to refer metaphorically to 'intelligence' as in brilliant idea or brilliant mind. This sense of brilliant expresses the metaphor KNOWING IS SEEING and its submappings SOURCES OF KNOWLEDGE ARE LIGHT SOURCES and INTELLIGENCE IS LIGHT-EMISSION, shown in Figure (6) below (LIGHT-EMISSION, which enables us to see objects, maps to INTELLIGENCE, which enables us to understand concepts). Since light-emission presupposes a light source, I will refer to these two submappings collectively as INTELLIGENCE IS LIGHT-EMISSION. ${ }^{3}$

\footnotetext{
${ }^{3}$ In accordance with the partial nature of metaphoric mappings, some instances of KNOWING IS SEEING do not involve the mapping INTELLIGENCE IS LIGHT-EMISSION. The observations in this section apply only to those usages of KNOWING IS SEEING in which the mapping is evident.
} 
(6) KNOWING IS SEEING and INTELLIGENCE IS LIGHT-EMISSION

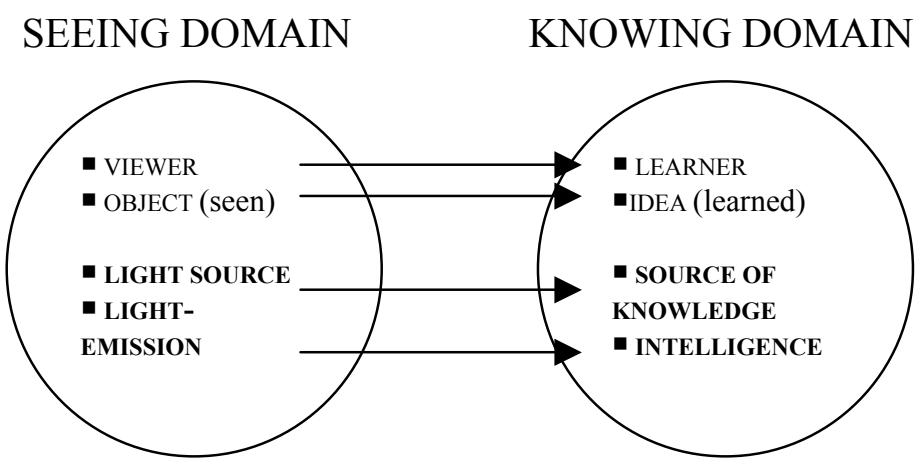

The mapping INTELLIGENCE IS LIGHT-EMISSION, like the metaphor HAPPINESS IS LIGHT, can be expressed by certain modifiers but not by others. We saw that brilliant expresses this submapping, as in brilliant mind. Like brilliant, the adjective dim can express INTELLIGENCE IS LIGHT-EMISSION, as in dimwit or dim child. The usage frequencies of these and other items are listed below.

Table 2. INTELLIGENCE IS LIGHT-EMISSION collocations

\begin{tabular}{|l|l|l|l|l|l|}
\hline Item & $\begin{array}{l}\text { Total } \\
\text { 'LIGHT' }\end{array}$ & Example & $\begin{array}{l}\text { Total } \\
\text { 'INTELLI- } \\
\text { GENCE' }\end{array}$ & Example & $\begin{array}{l}\text { Percent 'INTELLI- } \\
\text { GENCE' } \\
\text { (of total hits) }\end{array}$ \\
\hline brilliantly & $\mathbf{4 0}$ & shine brilliantly & $\mathbf{3 5}$ & reason brilliantly & $\mathbf{1 9 . 1}$ \\
\hline brilliant & $\mathbf{1 0 7 0}$ & brilliant sun & $\mathbf{1 7 9}$ & brilliant idea & $\mathbf{1 2 . 3}$ \\
\hline bright & $\mathbf{2 4 3 0}$ & bright jewel & $\mathbf{3 7 1}$ & bright student & $\mathbf{8 . 9}$ \\
\hline dim & $\mathbf{2 6 0}$ & dim star & $\mathbf{4}$ & dim child & $\mathbf{1 . 1}$ \\
\hline brightly & $\mathbf{3 8 2}$ & glow brightly & $\mathbf{0}$ & & $\mathbf{0}$ \\
\hline
\end{tabular}

Although a considerable percentage of the occurrences of brilliant reflect INTELLIGENCE IS LIGHT-EMISSION, other items, like sunny and dark, fail to express this metaphor even once. To explain the distinction between items like brilliant and items like sunny, let us return to the LIGHT_MOVEMENT frame evoked by brilliant, repeated as Figure (7) below.

(7) LIGHT_MOVEMENT frame (brilliant, bright, dim)

\begin{tabular}{|l|}
\hline - LIGHT SOURCE (EMITTER) \\
- LIGHT-EMISSION (BEAM) \\
a DEGREE (brightness) \\
$\ldots$. \\
\hline
\end{tabular}

Items like brilliant tend to modify nouns denoting the LIGHT SOURCE in this frame, as in brilliant star, brilliant flash, and brilliant sun. Conversely, items such as sunny and dark exhibited no collocations with LIGHT SOURCE nouns and presumably do not evoke the LIGHT_MOVEMENT frame. 
Karen Sullivan

The SEEING source domain of KNOWING IS SEEING, as in Figure (6) above, also includes a LIGHT SOURCE. This element maps to a SOURCE OF KNOWLEDGE in the KNOWING domain (a mapping evident in phrases like this book is illuminating or your answer shed light on the topic). The presence of this LIGHT SOURCE element indicates that the LIGHT_MOVEMENT frame is active in the SEEING source domain.

Now that we have identified LIGHT_MOVEMENT frame structure in the SEEING domain, centered around the submapping INTELLIGENCE IS LIGHT-EMISSION, we can make a prediction: Only lexical items that evoke the LIGHT_MOVEMENT frame, as brilliant does, will be chosen to express INTELLIGENCE IS LIGHTEMISSION.

In fact, the rest of the items under consideration support this generalization. Like brilliant, the item dim often literally refers to a light source, as in dim star, dim torch or dim lantern. This reference to LIGHT-EMISSION allows dim to refer metaphorically to INTELLIGENCE as in dim child.

Unlike brilliant, sunny does not modify light source nouns, but refers only to ambient sunlight. The item sunny could, in theory, be used image-metaphorically to describe a light source that resembles sunlight, as in ?sunny firelight or ? sunny lantern; but there were no examples of this type in the corpus. The item sunny does not typically evoke LIGHT_MOVEMENT and, as a result, is incompatible with INTELLIGENCE IS LIGHT-EMISSION.

Like sunny, the item dark was not found to modify potential light sources (?dark streetlight). In practice, dark seems to refer to a level of available light, not to an absence of light from a given source. Consequently dark does not refer to a lack of intelligence via INTELLIGENCE IS LIGHT-EMISSION.

The LIGHT SOURCE element in the LIGHT_MOVEMENT frame seems to determine adjectives' compatibility with INTELLIGENCE IS LIGHT-EMISSION, much in the same way that the LOCATION element in LOCATION_OF_LIGHT predicted compatibility with HAPPINESS IS LIGHT. This consistency between frames and mappings supports the idea that semantic frames shape items' metaphoric uses.

\subsection{The metaphoric and nonmetaphoric polysemies of bright}

Most of the adjectives we have examined (brilliant, dim, sunny and dark) work with either HAPPINESS IS LIGHT or INTELLIGENCE IS LIGHT-EMISSION, but not with both. The adjective bright is the exception. Alongside the metaphoric uses meaning 'cheerful', as in bright mood, we find collocations like bright idea and bright child, where bright means 'intelligent'.

The metaphoric polysemy of bright is put in perspective when we consider the diverse nonmetaphoric senses of the item. While one sense of bright evokes the LOCATION_OF_LIGHT frame, as in bright room etc., a second sense of bright evokes the LIGHT_MOVEMENT frame, as in bright fire, bright object and bright moon.

In accordance with the Invariance Principle, the frame evocation properties of the nonmetaphoric senses of bright are preserved in its metaphoric uses. The 
sense of bright in bright room can, as a result, extend to the metaphoric sense in bright mood via HAPPINESS IS LIGHT, whereas the sense in bright fire can extend to the metaphoric sense in bright idea via INTELLIGENCE IS LIGHT-EMISSION. None of the other adjectives share this polysemy, and as a result, only bright can express both HAPPINESS IS LIGHT and INTELLIGENCE IS LIGHT-EMISSION.

It is worth noting that although both bright and brilliant can express INTELLIGENCE IS LIGHT-EMISSION, bright denotes a lesser DEGREE of intelligence than brilliant. The adjective bright often refers to children or students, as in bright child, bright boy, or bright pupil. In contrast, brilliant is more likely to occur in brilliant engineer, brilliant scholar or brilliant scientist. This distinction shows that the values assigned to the DEGREE element in the LIGHT_MOVEMENT frame (in which brilliant involves a greater DEGREE of light-emission than bright) are carried over into the target domain, in which the adjectives denote differing DEGREES of intelligence.

\section{KNOWING IS SEEING and COMPREHENSIBILITY IS VISIBILITY}

The final set of examples I will discuss involve another submapping of KNOWING IS SEEING, COMPREHENSIBILITY IS VISIBILITY. Certain items that cannot refer to 'stupidity' or 'intelligence' nevertheless have metaphoric meanings related to KNOWING IS SEEING. For example, a dim idea normally means a 'vague' or 'uncertain' idea, not a 'stupid' one. The item dark similarly can refer to something 'unknown' or 'mysterious', as in a dark area in our understanding. Most dramatically, the adjective clear means 'obvious' or 'certain' 83 percent of the time as in a clear understanding, clear idea or a clear statement, and adverbial clearly means 'certainly' or 'obviously' 86 percent of the time, as shown below.

Table 3. COMPREHENSIBILITY IS VISIBILITY collocations

\begin{tabular}{|l|l|l|l|l|l|}
\hline ITEM & $\begin{array}{l}\text { TOTAL } \\
\text { 'LIGHT' }\end{array}$ & EXAMPLE & $\begin{array}{l}\text { TOTAL } \\
\text { 'COMPRE- } \\
\text { HENSIBILITY' }\end{array}$ & EXAMPLE & $\begin{array}{l}\text { PERCENT } \\
\text { 'COMPRE- } \\
\text { HENSIBILITY' } \\
\text { (of total hits) }\end{array}$ \\
\hline clearly & $\mathbf{3 8 1}$ & see clearly & $\mathbf{2 3 0 8}$ & reason clearly & $\mathbf{8 5 . 8}$ \\
\hline clear & $\mathbf{5 5 8}$ & clear image & $\mathbf{2 9 1 9}$ & clear statement & $\mathbf{8 2 . 8}$ \\
\hline dim & $\mathbf{2 6 0}$ & dim figure & $\mathbf{3 0}$ & dim idea & $\mathbf{8 . 7}$ \\
\hline dark & $\mathbf{4 3 4 0}$ & dark shape & $\mathbf{5 1}$ & $\begin{array}{l}\text { dark area of } \\
\text { understanding }\end{array}$ & $\mathbf{1 . 1}$ \\
\hline
\end{tabular}

Items like clear 'obvious/certain' are not based on the 'light-emission' mapping of KNOWING IS SEEING that is active in brilliant 'intelligent'. Instead, these uses focus on the sub-mappings IDEAS ARE OBJECTS and COMPREHENS-IBILTY (of an idea) IS VISIBILITY (of an object), shown in Figure (8). Because the latter mapping presupposes the former, I refer to these two submappings collectively as COMPREHENSIBILITY IS VISIBILITY. 
(8) KNOWING IS SEEING and COMPREHENSIBILITY IS VISIBILITY

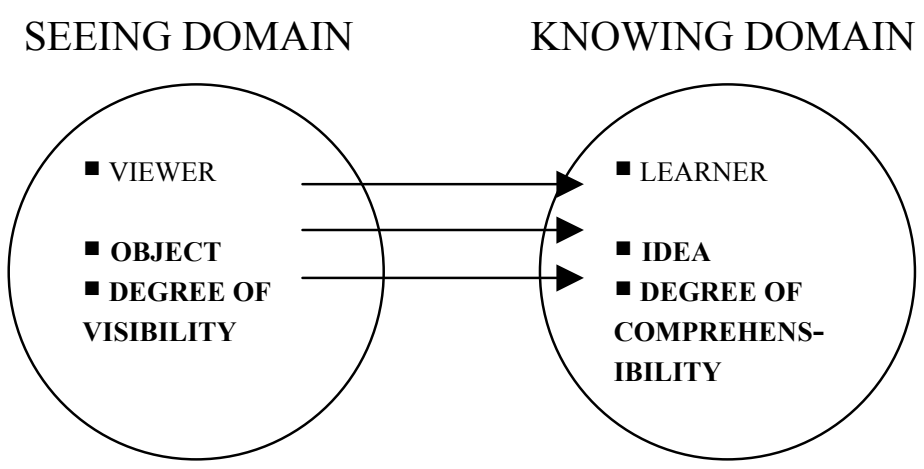

We know that items are not chosen to express COMPREHENSIBILITY IS VISIBILITY on the basis of the LIGHT_MOVEMENT frame, because, as we saw in the previous section, clear and dark do not evoke this frame.

Instead, the nonmetaphoric uses of the relevant items point to a different frame involved in COMPREHENSIBILITY IS VISIBILITY. Even though the item dim occurs in collocations denoting a light source (as in dim lantern), in fact dim more often refers to an object that is only vaguely or partially seen (dim shape, dim figure, or dim blur). These senses evoke a frame that includes a visible object and a level of visibility ascribed to that object. These elements may seem familiar, because we saw them in the LOCATION_OF_LIGHT frame (a frame that structures HAPPINESS IS LIGHT).

The LOCATION_OF_LIGHT frame, shown in Figure (3), is repeated in Figure (9) with a few changes in emphasis. Nouns modified by clear or dim denote the FIGURE element in the LOCATION_OF_LIGHT frame, rather than the LOCATION element evoked by nouns in phrases like bright room. Noun phrases like bright room denote a LOCATION (such as a 'room'), whereas noun phrases like clear outline denote the FIGURE that is visible in some LOCATION (such as a visible 'outline'). A related difference between Figure (3) and Figure (9) is that the DEGREE element in LOCATION_OF_LIGHT as evoked by clear, dim etc. refers specifically to the visibility of the FIGURE rather than the brightness of light at the LOCATION.

(9) LOCATION_OF_LIGHT frame (clear, dim, dark)

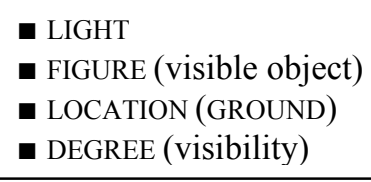

Just as the LOCATION element in LOCATION_OF_LIGHT is apparent in the source domain of HAPPINESS IS LIGHT, the FIGURE and DEGREE (of visibility) in this frame contribute structure to the source domain of KNOWING IS SEEING. The 


\section{Frame-Based Constraints on Lexical Choice in Metaphor}

element DEGREE (of visibility) maps from SEEING to KNOWING via DEGREE OF COMPREHENSIBILITY IS DEGREE OF VISIBILITY, as we saw in Figure (8).

As a result of this mapping, items like dim, which have nonmetaphoric uses referring to the VISIBILITY of a FIGURE (dim shape, dim outline) also allow metaphoric uses referring to the COMPREHENSIBILITY of an IDEA (dim memory, dim idea or dim awareness). Clear and dark also refer to the VISIBILITY of a FIGURE (clear footprint or dark shape), so these items can likewise refer metaphorically to COMPREHENSIBILITY (clear idea or the idiom dark horse).

These collocations show that COMPREHENSIBILITY IS VISIBILITY imposes different frame requirements on lexical choice than INTELLIGENCE IS LIGHTEMISSION, even though both are submappings of KNOWING IS SEEING. Since the LIGHT_MOVEMENT and LOCATION_OF_LIGHT frames are both part of the SEEING domain, certain submappings of KNOWING IS SEEING map structure from one frame, while different submappings map structure from the other.

\section{Adjectives vs. adverbs: brilliantly, clearly, brightly and sunnily}

Adverbs and adjectives that share a common root generally evoke the same frames, and as a result have the same range of metaphoric uses. For example, brilliant and brilliantly express INTELLIGENCE IS LIGHT-EMISSION 19.1\% and $12.3 \%$ of the time, respectively, as in Table 2; and clear and clearly express COMPREHENSIBILITY IS VISIBILITY $82.8 \%$ and $85.8 \%$ of the time, as in Table 3. Once again, similarities in frame structure lead to similar metaphoric uses.

Discrepancies between adjectives and adverbs can usually be attributed to factors other than frame structure. For example, brightly differs from bright in that brightly is not used in INTELLIGENCE IS LIGHT-EMISSION (Table 2). This discrepancy is part of a more general trend in which roots referring to 'intelligence' are less likely to be used as adverbs than roots referring to 'cheerfulness'. For example, the collocation intelligent $N(\mathrm{n}=916)$ is twice as common in the BNC than cheerful $N(\mathrm{n}=441)$, yet adverbial intelligently $V(\mathrm{n}=38)$ is rare compared to cheerfully $V(\mathrm{n}=151)$.

The adverb sunnily presents another case of adverb/adjective difference. Although sunny refers to HAPPINESS only $5.6 \%$ of the time, the lone instance of sunnily refers to HAPPINESS (Table 1). The nonoccurrence of literal sunnily is due to a combination of two factors: First, just as the adjective sunny does not normally modify nouns denoting light sources other than the sun (section [4]), sunnily rarely modifies verbs denoting light-emission produced by light sources other than the sun (?the firelight burned sunnily or ? the lantern shone sunnily). Second, sunnily is redundant in describing light-emission that actually is from the sun, as in ?the sun shone sunnily or ?the sunlight gleamed sunnily. These two restrictions conspire to rule out most nonmetaphoric uses of sunnily.

In the absence of factors such as those at work in brightly and sunnily, adverbs seem to share the metaphoric uses of their adjectival counterparts. The previous sections have shown that adjectives that evoke the same frames (such as bright, sunny and dark, which all evoke LOCATION_OF_LIGHT) have the same metaphoric 
Karen Sullivan

uses (such that bright, sunny and dark all express HAPPINESS IS LIGHT). Apparently, adjectives and adverbs which evoke the same frames likewise have similar metaphoric uses. This suggests that items' frame evocation is a more important factor than lexical category in determining metaphoric usages.

\section{Conclusion}

There seems to be a certain logic behind the choice of lexical items in expressing metaphor. Acknowledging this logic is an important step for conceptual metaphor theory, because understanding the regularities of lexical choice in metaphor will improve metaphor theorists' control over language as a data source. Furthermore, the central role of frame semantics in metaphoric language should draw attention to the significance of frames in conceptual metaphor and the necessity for an extended Invariance Principle.

The correlations noted in this paper also suggest three new avenues for research: First, the role of frames in metaphoric extension needs to be tested in conceptual domains other than LIGHT and SEEING. Second, the frame evocation properties of items belonging to other lexical categories (particularly verbs) remain to be examined. And finally, we should look for other forces that help shape lexical choice in metaphor. These will include stylistic concerns (such as the redundancy issue affecting sunnily), which should be sorted out from considerations such as frame compatibility. We have seen in this paper that lexical choice in metaphor is anything but arbitrary. However, it will take time, ingenuity, and a great deal of further research to unravel the complexity of factors at work in metaphoric language.

\section{References}

Fillmore, Charles J. 1982. Frame Semantics. In The Linguistic Society of Korea, ed., Linguistics in the Morning Calm, 111-137. Seoul: Hanshin Publishing Co.

Kövecses, Zoltan. 2002. Metaphor. Oxford: Oxford University Press.

Lakoff, George 1993. The Contemporary Theory of Metaphor. In Andrew Ortony, ed. Metaphor and Thought, $2^{\text {nd }}$ edition, 202-251. Cambridge: Cambridge University Press.

Lakoff, George and Mark Johnson 1980. Metaphors We Live By. Chicago: The University of Chicago Press.

Sweetser, Eve 1990. From etymology to pragmatics: Metaphorical and cultural aspects of semantic structure. Cambridge: Cambridge University Press. 


\section{Frame-Based Constraints on Lexical Choice in Metaphor}

Karen Sullivan

University of Queensland

School of Languages and Comparative Cultural Studies

Gordon Greenwood

QLD 4072

Australia

ksull@uq.edu.au 